\title{
Erratum: Edge-induced topological phase transition of the quantum Hall state at half filling [Phys. Rev. B 99, 161108(R) (2019)]
}

Bo Yang, Na Jiang, Xin Wan, Jie Wang, and Zi-Xiang Hu

(Q) (Received 29 April 2019; published 13 May 2019)

DOI: 10.1103/PhysRevB.99.209901

This paper was published online on 8 April 2019 with a mislabeled legend for Fig. 2(a) on p. 3. The legend for Pf and APf was reversed. The corrected figure is attached here.
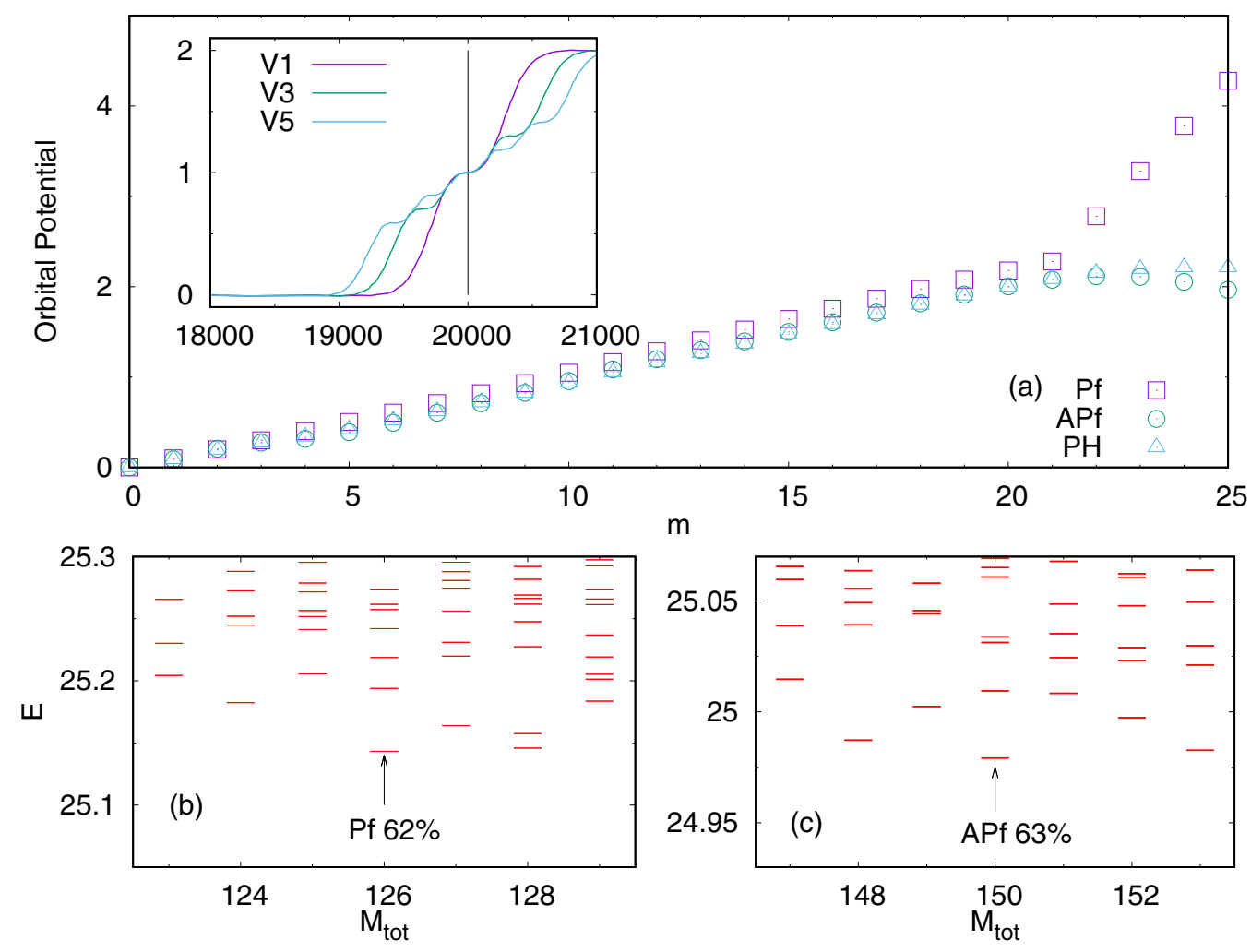

FIG. 2. 